\title{
Effect of Fascia Penetration in Lateral Femoral Cutaneous Nerve Conduction
}

\author{
Mi-Jeong Yoon, MD, PhD, Hye Min Park, BD, Sun Jae Won, MD, PhD \\ Department of Rehabilitation Medicine, Yeouido St. Mary's Hospital, \\ College of Medicine, The Catholic University of Korea, Seoul, Korea
}

Objective To evaluate the effect of fascia penetration and develop a new technique for lateral femoral cutaneous nerve (LFCN) conduction studies based on the fascia penetration point (PP) identified using ultrasound.

Methods The fascia PP of the LFCN was localized in 20 healthy subjects, and sensory nerve action potentials (SNAPs) were obtained at four different stimulation points $-2 \mathrm{~cm}$ proximal to the $\mathrm{PP}(2 \mathrm{PPP}), \mathrm{PP}, 2 \mathrm{~cm}$ distal to the PP (2DPP), and $4 \mathrm{~cm}$ distal to the PP (4DPP). We compared the stimulation technique based on the fascia penetration point (STBFP) with the conventional technique.

Results The SNAP amplitude of the LFCN was significantly higher when stimulation was performed at the PP and 2DPP than at other stimulation points. Using the STBFP, SNAP responses were elicited in 38 of 40 legs, whereas they were elicited in 32 of 40 legs using the conventional technique $(\mathrm{p}=0.041)$. STBFP had a comparable SNAP amplitude and slightly delayed negative peak latency compared to the conventional technique. In terms of the time required, the time spent on STBFP showed a more consistent distribution than the time spent on the conventional technique (two-sample Kolmogorov-Smirnov test, $\mathrm{p}<0.05$ ).

Conclusion SNAP of the LFCN significantly changed near the fascia PP, and stimulation at PP and at 2DPP provided high amplitudes. STBFP can help increase the response rate and ensure stable and consistent procedure time of the LFCN conduction study.

Keywords Electromyography, Methods, Neural conduction, Physiology, Ultrasonography, Femoral nerve, Diagnostic imaging, Humans

\section{INTRODUCTION}

The lateral femoral cutaneous nerve (LFCN) is derived from the posterior divisions of the L2 and L3 spinal nerves. The nerve originates within the psoas major and passes across the iliac fossa beneath the iliac fascia, traveling toward the medial side of the anterior superior iliac spine (ASIS). It passes behind or through the iliac liga-

Received February 12, 2020; Revised April 9, 2020; Accepted May 19, 2020; Published online December 31, 2020 Corresponding author: Sun Jae Won

Department of Rehabilitation Medicine, Yeouido St. Mary's Hospital, College of Medicine, The Catholic University of Korea, 10 63(yuksam)-ro, Yeongdeungpo-gu, Seoul 07345, Korea. Tel: +82-2-3779-1064, Fax: +82-2-3779-2075, E-mail: gstinfog@catholic.ac.kr

ORCID: Mi-Jeong Yoon (https://orcid.org/0000-0003-0526-6708); Hye Min Park (https://orcid.org/0000-0003-0818-9410); Sun Jae Won (https://orcid. org/0000-0002-9057-3747).

(c) This is an open-access article distributed under the terms of the Creative Commons Attribution Non-Commercial License (http://creativecommons.org/ licenses/by-nc/4.0) which permits unrestricted noncommercial use, distribution, and reproduction in any medium, provided the original work is properly cited. Copyright (C) 2020 by Korean Academy of Rehabilitation Medicine 
ment and enters the thigh with highly variable anatomical courses [1]. In the thigh, the nerve penetrates the fascia lata and divides into terminal branches to innervate the anterolateral skin of the thigh [2].

Meralgia paresthetica is a condition characterized by tingling, numbness, and burning pain in the anterolateral thigh and is usually caused by damage to the LFCN [3]. Sensory nerve conduction studies help identifying LFCN lesions. The procedure involves stimulating the LFCN $1 \mathrm{~cm}$ medial to the ASIS and recording at a distance of $12-16 \mathrm{~cm}$ along the line extending from the ASIS to the lateral border of the patella, to obtain the sensory nerve action potential (SNAP) response of the LFCN [4]. However, this conventional LFCN conduction technique has a limitation owing to its deep position and anatomical variations in the inguinal area and proximal leg [5]. To obtain accurate SNAPs of the LFCN, various techniques have been developed, such as ultrasound for the localization of the LFCN [6,7], subdermal needle recording [8], and assessment of LFCN conduction in the distal thigh [5].

Recently, several studies reported the effect of fascia on conduction studies of cutaneous nerves, suggesting that stimulation based on the fascia penetration point (PP) can produce more accurate and reliable SNAP results $[9,10]$. According to previous studies, SNAP amplitude was the highest when the nerve was stimulated immediately after penetrating the fascia $[9,10]$. Increased depth from the skin to the nerve, low conductivity of fat, and large electrical conduction activity of the fascia itself can interfere with nerve action potentials and decrease SNAP amplitudes in sensory nerve conduction. Similar to other cutaneous nerves, the LFCN is located below the fascia of the iliacus muscle and penetrates the fascia in the thigh, becoming superficial. Therefore, we hypothesized whether stimulation around the fascia PP of the LFCN can improve the measurement of nerve conduction study (NCS) parameters. To this end, we aimed to identify the effect of fascia penetration in LFCN conduction study and to compare the stimulation technique based on the fascia penetration point (STBFP) with the conventional technique.

\section{MATERIALS AND METHODS}

This study was approved by the Institutional Review Board of Yeouido St. Mary's Hospital (No. SC18EESI0144).
Healthy subjects were recruited, and written informed consent was obtained. The study was a randomized controlled comparison between the conventional LFCN conduction technique and STBFP.

\section{Subjects}

Forty healthy subjects were enrolled in this study. The exclusion criteria included aged $<20$ or $>65$ years; history of diabetes; neuromuscular diseases involving the extremities, such as peripheral neuropathy or radiculopathy; or history of hip or thigh surgery. Forty subjects were randomly assigned to either the conventional LFCN conduction group utilizing ASIS as a landmark or to the STBFP group, by employing a random allocation website (http://www.randomization.com/) and blinding to group assignment. Demographic data were collected at the beginning of the study. Leg length was defined as the distance between the ASIS and the medial malleolus in the supine position.

\section{Ultrasonography}

In the STBFP group, ultrasonography was performed before sensory nerve conduction by a skilled physiatrist with more than 10 years' experience in neuromusculoskeletal ultrasonography. To identify the course of the lateral femoral cutaneous nerve, the subject was placed in the supine position. The probe was then placed around the ASIS. Tracing was initiated at a site inferior to the ASIS. First, the sartorius muscle was identified. The LFCN runs consistently between the fascia lata and the sartorius muscle (underneath the fascia lata and superficial to the sartorius muscle) in the proximal anterolateral thigh area. By tracing the LFCN cephalo-caudally, the point where the LFCN penetrated the fascia lata was identified and defined as PP. In some subjects, it was challenging to visualize the LFCN clearly when it was penetrating the fascia lata and its depth changed rapidly around the ASIS. The PP was marked at the most proximal point where the LFCN could be clearly visualized above the fascia lata. The course of the LFCN was marked on the skin with ultrasound tracing of the main branch of the LFCN distally (Fig. 1). The time from the initial contact of the ultrasound transducer with the skin to marking the course of the LFCN was measured and recorded. The distance between the PP and the inferior margin of the ASIS was measured. The cross-sectional area of the LFCN 


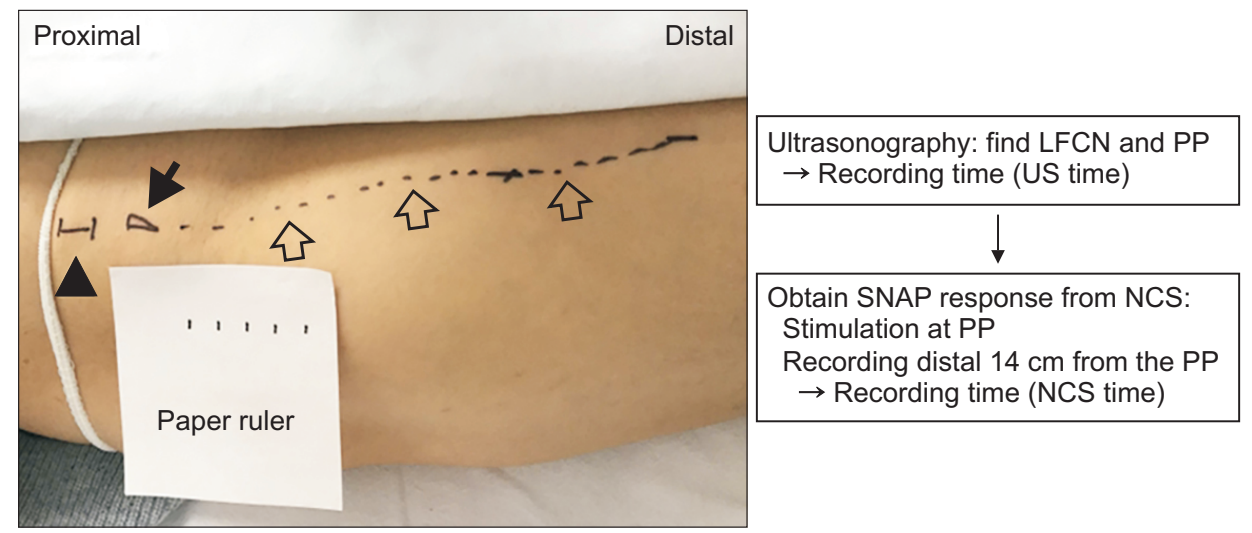

Fig. 1. The study protocol of the stimulation technique based on the fascia penetration point. (1) Find LFCN using sonography and mark the fascia PP (filled arrow), the nerve course (unfilled arrow) and ASIS (black arrowhead) on the surface of right thigh as shown in left image. (2) Evaluate the time spent on US. (3) Stimulate the fascia PP and obtain SNAP at recording site distal $14 \mathrm{~cm}$ from the stimulation point. (4) Evaluate the time spent on NCS and VAS. The white paper ruler (scale drawn on white paper at $1 \mathrm{~cm}$ intervals) is placed alongside the nerve course marking. Left side of the image is proximal. LFCN, lateral femoral cutaneous nerve; PP, penetration point; ASIS, anterior superior iliac spine; US, ultrasonography; SNAP, sensory nerve action potential; NCS, nerve conduction study; VAS, visual analogue scale.
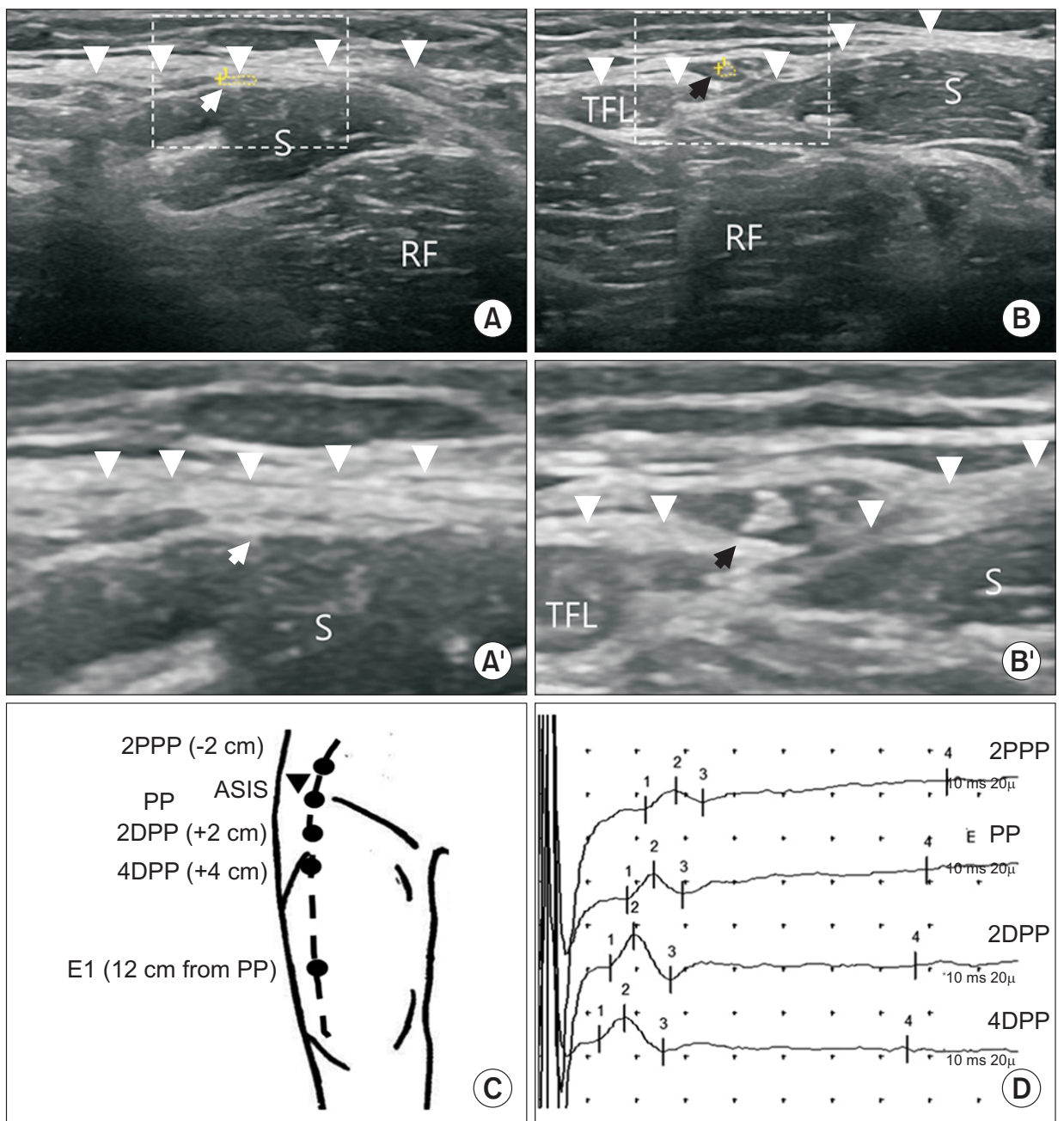

Fig. 2. Sonography and sensory nerve conduction study of the lateral femoral cutaneous nerve. (A) Lateral femoral cutaneous nerve (dotted circle with white arrow) beneath the fascia lata (arrowheads). (B) Lateral femoral cutaneous nerve (dotted circle with black arrow) just after penetrating the fascia. (A') Enlarged image of the part indicated by a dotted rectangle in panel A. (B') Enlarged image of the part indicated by a dotted rectangle in panel B. (C) Stimulation point based on the PP in the lateral femoral cutaneous nerve conduction study. (D) Sensory nerve action potentials at different stimulation points. $\mathrm{RF}$, rectus femoris muscle; TFL, tensor fascia lata muscle; $\mathrm{S}$, sartorius muscle; $2 \mathrm{PPP}, 2 \mathrm{~cm}$ proximal to the penetration point; $\mathrm{PP}$, the penetration point; 2DPP, $2 \mathrm{~cm}$ distal to the penetration point; $4 \mathrm{DPP}, 4 \mathrm{~cm}$ distal to the penetration point; ASIS, anterior superior iliac spine. 
was measured at the location immediately after passing through the fascia (Fig. 2A, 2B, 2A', 2B'). After ultrasonography, subjects were transferred to the electromyography room for NCS.

\section{Sensory nerve conduction study}

Sensory NCSs were performed by one electromyographer in both groups, specifically blinded to the time spent on ultrasonography in the STBFP group. LFCN conduction was performed bilaterally. In the conventional LFCN conduction group, the El electrode was placed $14 \mathrm{~cm}$ distal to the ASIS along an imaginary line between the ASIS and the lateral border of the patella. The E2 electrode was always placed $4 \mathrm{~cm}$ distal to the E1 in this study. Stimulation was performed with a stimulator placed $1 \mathrm{~cm}$ medial to the ASIS until supramaximal electrical stimulations were obtained. Ten responses were elicited after supramaximal stimulation was achieved. The largest SNAP amplitude (peak to baseline amplitude) was selected, and its negative peak latency was measured. Time spent on procedures and visual analog scale (VAS) scores for pain were recorded. In the STBFP group, the E1 electrode was placed $14 \mathrm{~cm}$ distal to the PP on the LFCN course and the stimulation was performed at the PP. The SNAP amplitudes, negative peak latencies, procedure time, and VAS scores for pain were obtained for comparison with the conventional technique. The time for STBFP was the summation of the time spent on ultrasonography and on the sensory NCS. After measuring the time, the fascia penetration effect was evaluated: the El electrode was placed $12 \mathrm{~cm}$ distal to the PP and the LFCN was stimulated at four different sites, namely, at $2 \mathrm{~cm}$ proximal to the PP (2PPP), at the PP, at $2 \mathrm{~cm}$ distal to the PP (2DPP), and at $4 \mathrm{~cm}$ distal to the PP (4DPP) (Fig. 2C, 2D).

\section{Statistical analysis}

Variable distributions were assessed using the Kolmogorov-Smirnov test for normality. Normally distributed variables are presented as mean \pm standard deviation, whereas non-normally distributed variables are presented as median and interquartile range (IQR). Differences between the two groups were compared using Student t-test for parametric data and the Mann-Whitney U test for non-parametric data. Fisher exact test was used to compare responses between the conventional technique and STBFP. Two-sample Kolmogorov-Smirnov test was used to compare the overall distribution of the time spent on the two techniques. The Kruskal-Wallis test was used to analyze the differences in negative peak latencies and amplitudes of LFCN SNAPs at 2PPP, PP, 2DPP, and 4DPP. The Mann-Whitney test with Bonferroni correction was used for post-hoc analyses with $\mathrm{p}<0.0083(=0.05 / 6)$ considered as a significant difference; $\mathrm{p}<0.05$ was considered significant (except with Bonferroni correction). All statistical analyses were verified by a statistical consultant.

\section{RESULTS}

The demographics of the 40 subjects are shown in Table 1. In the STBFP group, the mean cross-sectional area of the LFCN at the PP was $1.50 \pm 0.55 \mathrm{~mm}^{2}$ and the mean distance between the LFCN at the PP and the inferior margin of the ASIS was $1.60 \pm 1.29 \mathrm{~cm}$ (range, $0.50-6.00 \mathrm{~cm}$ ) distal to the ASIS.

Table 1. Characteristics of the subjects studied

\begin{tabular}{lccc}
\hline & Conventional $^{\text {a) }}(\mathbf{n = 2 0})$ & STBFP $^{\mathbf{b})}(\mathbf{n}=\mathbf{2 0})$ & p-value \\
\hline Sex, female & 19 & 19 & \\
Age $(\mathrm{yr})$ & $49.00(12.75)$ & $51.00(11.75)$ & 0.968 \\
Body weight $(\mathrm{kg})$ & $55.50(12.65)$ & $56.50(12.38)$ & 0.547 \\
Height $(\mathrm{cm})$ & $160.12 \pm 5.24$ & $161.71 \pm 5.62$ & 0.389 \\
BMI $\left(\mathrm{kg} / \mathrm{m}^{2}\right)$ & $22.76 \pm 2.80$ & $22.42 \pm 2.18$ & 0.653 \\
Leg length $(\mathrm{cm})$ & $77.30 \pm 3.73$ & $79.00 \pm 4.02$ & 0.173 \\
\hline
\end{tabular}

Values are presented as median (interquartile range) or mean \pm standard deviation.

STBFP, stimulation technique based on the fascia penetration point; BMI, body mass index.

${ }^{\text {a) }}$ Stimulation $1 \mathrm{~cm}$ medial to the anterior superior iliac spine.

${ }^{\text {b) }}$ Stimulation based on fascia penetration.

No significant difference between the conventional and STBFP groups by the Mann-Whitney U test or Student t-test. 
Table 2. SNAP amplitudes, latencies, no response, time spent, and VAS scores in comparison of the two techniques

\begin{tabular}{lccc}
\hline & Conventional & STBFP & p-value \\
\hline Recording $(\mathrm{cm})$ & 14 & 14 & $0.006^{* \mathrm{~b})}$ \\
Peak latency $(\mathrm{ms})$ & $2.62 \pm 0.18$ & $2.75 \pm 0.21$ & $0.164^{\mathrm{b})}$ \\
Amplitude $(\mu \mathrm{V})$ & $6.97 \pm 2.16$ & $7.68 \pm 2.00$ & $0.041^{* \mathrm{~d})}$ \\
No response & & & \\
Subjects $(\mathrm{n}=20)$ & 4 & 2 & $0.419^{\mathrm{c})}$ \\
Legs $(\mathrm{n}=40)$ & 8 & $197.50(56.75)$ & \\
Time $(\mathrm{s})$ & $178.50(225.50)$ & $125.55 \pm 36.81$ & $0.244^{\mathrm{b})}$ \\
Ultrasonography $^{\mathrm{a})}(\mathrm{s})$ & - & $3.90 \pm 1.61$ & \\
\hline VAS score & $4.52 \pm 1.74$ & & \\
\hline
\end{tabular}

Values are presented as mean \pm standard deviation or median (interquartile range).

Data in the table are the values excluding the case of no response, and peak latency represents the negative peak latency.

VAS, visual analog scale; STBFP, stimulation technique based on the fascia penetration point.

${ }^{\text {a) }}$ Time spent finding the fascia PP of the lateral femoral cutaneous nerve using ultrasonography

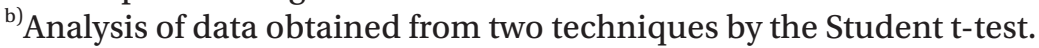

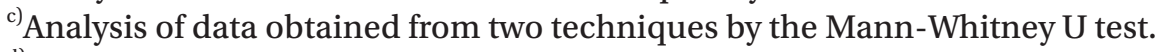

${ }^{\text {d) } A n a l y s i s ~ o f ~ F i s h e r ~ e x a c t ~ t e s t . ~}$

${ }^{*} \mathrm{p}<0.05$.

The results of the sensory NCS of the LFCN obtained from the two techniques are shown in Table 2. Using the conventional technique, 4 of 20 subjects showed no SNAP response in both the legs, whereas using STBFP, 2 of 20 subjects showed no SNAP response in only one leg. Statistical analysis showed that the response rate of STBFP was significantly higher than that of the conventional technique $(\mathrm{p}=0.041)$. The mean SNAP amplitude in the STBFP group was slightly higher than that in the conventional conduction group, although there was no significant difference between the two groups. The negative peak latency measured by STBFP was delayed by a small difference compared with that measured by the conventional technique. The median time spent in the STBFP group (the time from the placement of the ultrasound transducer on the skin to marking the course of the LFCN using ultrasound + time taken to obtain the LFCN SNAP) was 197.50 seconds (IQR, 56.75 seconds), which was comparable to that of the conventional conduction group (median time, 178.50 seconds; IQR, 225.50 seconds). However, a histogram of the time spent on the two techniques showed that STBFP took a more consistent time than the conventional technique (Fig. 3). The time spent on the conventional technique was variable (60.00-989.00 seconds), but the time spent on STBFP was consistent (126.00-345.00 seconds). The overall distributions of the

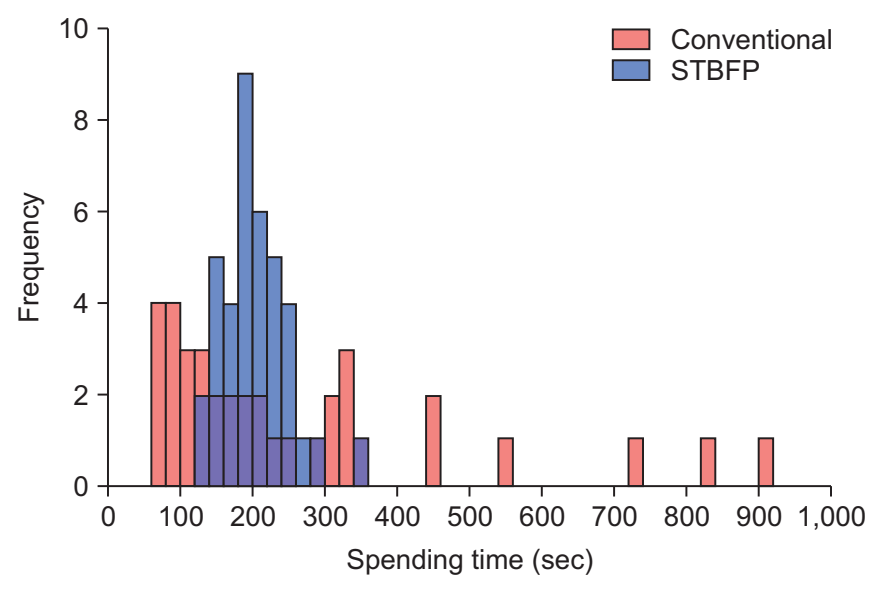

Fig. 3. Comparison of time spent on conventional technique and STBFP. Histogram shows spending time of two techniques, conventional technique (red) and STBFP (blue). The distribution of two histograms are significantly different according to the two-sample KolmogorovSmirnov test $(\mathrm{D}=0.342$, $\mathrm{p}$-value $=0.0173) . \mathrm{STBFP}$, stimulation based on fascia penetration.

time spent on the two techniques were significantly different (two-sample Kolmogorov-Smirnov test, $\mathrm{p}<0.05$ ). There were no significant differences in the VAS scores for pain during NCS $(4.52 \pm 1.74$ vs. $3.90 \pm 1.61)$ between the two groups.

SNAPs of the LFCN obtained from the four different 
Table 3. Sensory nerve conduction study of the lateral femoral cutaneous nerve based on PP

\begin{tabular}{lccccc}
\hline & 2PPP & PP & 2DPP & 4DPP & p-value \\
\hline Peak latency $(\mathrm{ms})$ & $2.75(0.36)$ & $2.45(0.25)$ & $2.10(0.20)$ & $1.85(0.19)$ & $<0.001^{*}$ \\
Amplitude $(\mu \mathrm{V})$ & $6.50(1.88)$ & $7.75(2.70)$ & $8.20(2.80)$ & $6.80(3.40)$ & $<0.001^{*}$ \\
\hline
\end{tabular}

Values are presented as median (interquartile range).

Peak latency represents the negative peak latency.

$\mathrm{PP}$, penetration point; $2 \mathrm{PPP}, 2 \mathrm{~cm}$ proximal to the penetration point; $2 \mathrm{DPP}, 2 \mathrm{~cm}$ distal to the penetration point; 4DPP, $4 \mathrm{~cm}$ distal to the penetration point.

${ }^{*} \mathrm{p}<0.001$ by Kruskal-Wallis test, significant differences among groups.

(A)

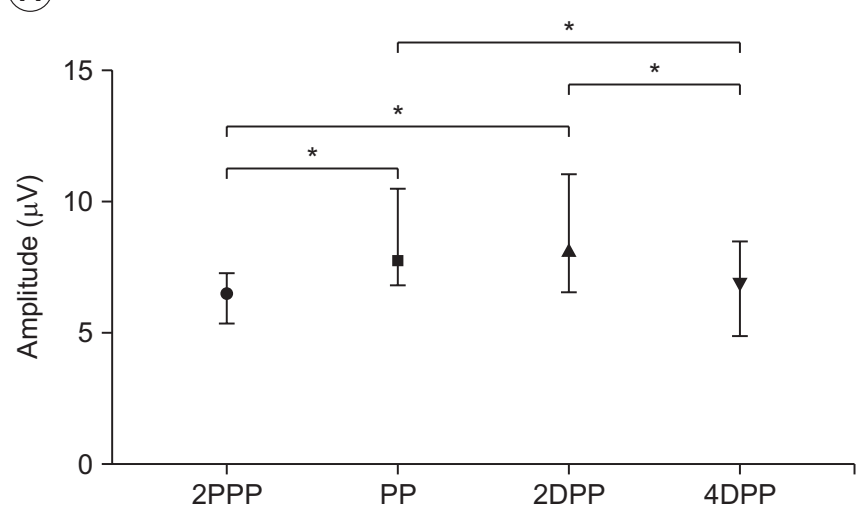

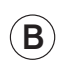

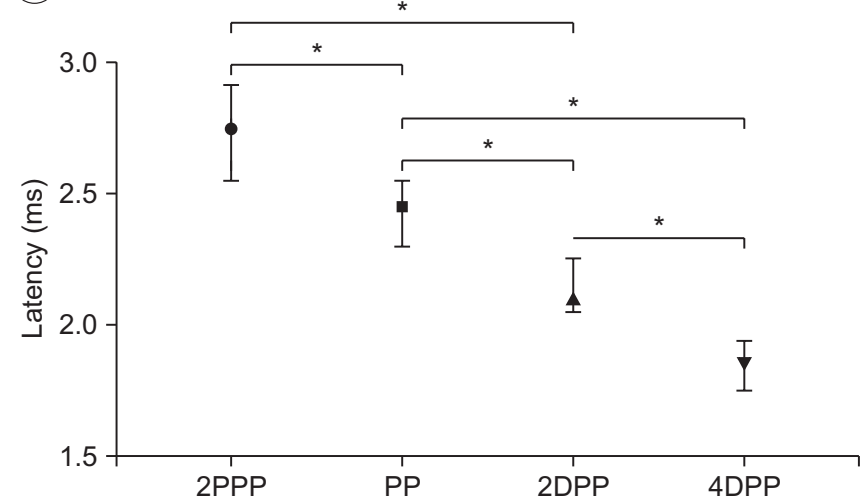

Fig. 4. Comparison of parameters between four different stimulation points based on the PP in the lateral femoral cutaneous nerve conduction study. (A) SNAP amplitude and (B) SNAP negative peak latency at each stimulation point as median and interquartile range. ${ }^{*} \mathrm{p}<0.0083$ comparison by post-hoc analysis (Mann-Whitney U test with Bonferroni correction), significant differences between groups. SNAP, sensory nerve action potential; $2 \mathrm{PPP}, 2 \mathrm{~cm}$ proximal to the penetration point; $\mathrm{PP}$, penetration point; 2DPP, $2 \mathrm{~cm}$ distal to the penetration point; $4 \mathrm{DPP}, 4 \mathrm{~cm}$ distal to the penetration point.

stimulation sites based on the fascia PP are shown in Fig. 2D. There were significant differences between the $2 \mathrm{PPP}$, PP, 2DPP, and 4DPP sites in terms of negative peak latencies and amplitudes ( $\mathrm{p}<0.001$ with Kruskal-Wallis test) (Table 3). Post-hoc analysis using the Mann-Whitney U test with Bonferroni correction showed that the SNAP amplitudes at the PP and 2DPP were significantly higher than those at the other test points (Fig. 4A). However, there was no significant difference in the SNAP amplitude between the PP and 2DPP sites. Negative peak latency was different at all four points (Fig. 4B).

\section{DISCUSSION}

Our study shows that fascia penetration has a significant effect on sensory nerve conduction of the LFCN. Because the fascia conducts electricity better than subcutaneous fat, the electrical stimulation can be transmitted unequally around the fascia, causing interference of the nerve action potentials and reducing the SNAP amplitude [10]. Furthermore, in the abdominal and inguinal area, there is usually a significant amount of peritoneal fat and connective tissue around the LFCN, which may interfere with the conduction of electrical activity [11]. Therefore, it is plausible that stimulation of the nerve at the point piercing the fascia and positioning beneath the skin can obtain larger SNAP amplitudes. To test our hypothesis, we identified the fascia PP of the LFCN using ultrasonography and stimulated the LFCN at four different stimulation sites based on the fascia PP. The results showed that LFCN stimulation between the PP and 2DPP sites had significantly higher SNAP amplitudes than those at other stimulation points, suggesting that the fascia PP should be considered in LFCN conduction studies. Previous studies have reported the effects of fascia penetration on several nerves $[9,10]$, but the LFCN has not 
been studied. This is the first study to evaluate the fascia penetration effect in nerve stimulation for LFCN conduction.

Different anatomical variations of the LFCN are major obstacles in obtaining consistent results in nerve conduction studies. Several techniques have been developed for obtaining LFCN SNAPs. Spevak and Prevec [5] stimulated the LFCN on the thigh with standard surface bipolar electrodes placed $6-10 \mathrm{~cm}$ below the ASIS, where the nerve usually lies underneath the skin, yielding low SNAP amplitudes with small variability. Two studies proposed a method of measuring the LFCN SNAP by visualizing the nerve using ultrasound and placing a surface electrode on it [6,7]. Deimel et al. [8] reported that the ultrasoundguided near-nerve needle recording method for LFCN conduction studies could be helpful when the surface recording of the LFCN elicits no response. Previously studied techniques mainly focused on medial or lateral positioning of the stimulation electrode, only at the level of the ASIS or the inguinal ligament $[4,12]$. Our study suggests that the proper stimulation point, considering the fascia PP of the LFCN, is approximately $1-3 \mathrm{~cm}$ distal to the ASIS. The STBFP LFCN conduction technique, developed by applying this electrophysiological effect of fascia penetration, may be helpful in improving the quality of LFCN conduction. To determine whether STBFP can obtain more accurate SNAP parameters than the previous ultrasound-guided conduction technique, further comparative studies using the two techniques are necessary.

The STBFP may be more beneficial than conventional LFCN conduction. First, the STBFP was found to have a higher detection rate for the SNAP response of the LFCN; the STBFP obtained SNAPs in 38 of 40 legs, whereas the conventional technique obtained SNAPs in 32 of 40 legs. Fisher exact test showed that the SNAP response strongly correlated with STBFP, and the odds ratio was 5.33. Furthermore, in terms of time, the ultrasound detection of the LFCN did not require a substantial amount of time. The average time for localization of the LFCN using ultrasound was approximately 2 minutes, and the time to obtain a SNAP response was approximately 80 seconds. Although the total time taken by STBFP was not significantly different compared with the conventional technique, variability of the time spent remarkably decreased when using STBFP. A consistent and stable procedure time when using STBFP can reduce patient discomfort

\section{during NCS.}

In this study, ultrasonography revealed that the LFCN pierces the fascia lata at an average of $1-3 \mathrm{~cm}$ distal to the inferior margin of the ASIS. The fascia PP in this study showed less variation and more proximal location, compared with previous cadaveric studies, which demonstrated that the LFCN usually pierces the fascia lata at approximately $5-10 \mathrm{~cm}$ distal to the inguinal ligament $[3,13,14]$. Ray et al. [15] reported that the mean distance between the ASIS and the point where the anterior division of the LFCN pierced the deep fascia of the thigh to become cutaneous was $18.84 \pm 8.58 \mathrm{~cm}$ (range, 3-30.5 $\mathrm{cm}$ ), with significant variations. The distal superficial branches of the LFCN pierce the fascia lata at $9.5 \pm 4.9 \mathrm{~cm}$ (range, 3.1-23.2 cm) distal to the inguinal ligament [16]. As shown in previous studies, the fascia PP is extremely variable, and most studies have examined Western populations using cadaveric studies. In this study, most subjects were Asian women. Different leg lengths and ethnicities might be a cause of the different results. Furthermore, the exact point at which the LFCN becomes superficial in the thigh may vary, like the anatomical variation of the LFCN course.

On comparison of the LFCN SNAP parameters of the conventional technique with those of STBFP, the negative peak latency of the conventional technique was slightly shorter than that of STBFP, although the distance from the stimulation electrode to the recording electrode was the same (Table 2). Because the LFCN runs deep under the inguinal ligament and its depth is extremely variable [17], its depth can change rapidly as the nerve passes around the ASIS. Therefore, when nerve stimulation is performed near the ASIS using the conventional technique, the nerve may not be stimulated just below the stimulation electrode, but at a slightly distal point where the nerve becomes superficial, in some cases. This would have the effect of shortening the actual distance between the stimulation point and the recording electrode, thereby reducing the negative peak latency in the conventional technique.

In some subjects, the fascia PP was quite distal, making it difficult to reliably track the nerve at a distal site 8-10 $\mathrm{cm}$ beyond the PP. For the comparison study of the SNAP response obtained by changing the stimulation site based on the fascia PP (Table 3), we placed the recording electrode $12 \mathrm{~cm}$ distal rather than $14 \mathrm{~cm}$ distal to the PP for 
stable recording. In the 4DPP stimulation, the distance between the stimulation electrode and the recording electrode was too short $(8 \mathrm{~cm})$; thus, stimulus artifacts occurred, and the amplitude was reduced. Because the SNAP amplitude of the LFCN was relatively small and subcutaneous fat required strong stimulations for LFCN conduction, stimulus artifacts could not be completely avoided at the 4DPP stimulation, despite strict adherence to the principles for reducing stimulus artifacts, including anodal rotation.

As there were steps involving examiners, such as ultrasound and nerve conduction, it was challenging to design a study that maintained objectivity and avoided bias. To directly compare STBFP with the conventional technique, it may be appropriate to perform the two techniques simultaneously on one subject, but differences in skill between examiners can affect the results. If one examiner performs the two techniques sequentially on one subject, there is a risk of performance bias affected by the order of the procedures. Therefore, we thought that performing each technique on two subjects assigned via rigorous random allocation would reduce the performance bias caused by ultrasound and NCS examiners and would produce the most objective study.

Our study had several limitations. First, we did not examine meralgia paresthetica patients. The diagnostic application of STBFP to meralgia paresthetica patients requires further validation and comparative studies in normal control and symptomatic patients. Second, although the nerve location was determined using ultrasound for the LFCN conduction study, there were two subjects with no response, and the cause of the lack of response was not identified. The proportion of subjects with no SNAP response in our results is similar to that reported in previous studies on LFCN conduction $[6,7]$. In the ultrasound-guided NCS by Boon et al. [6], 1 in 50 subjects showed no response, and in the study by Park et al. [7], 1 in 29 subjects showed no response. Further studies are required to evaluate whether the SNAP response can be evoked in unresponsive subjects by applying a near-needle stimulation technique, as shown in a study by Boon et al. [6]. Meanwhile, we attempted to include only healthy subjects in this study through history taking, but it is possible that patients with asymptomatic lateral femoral cutaneous neuropathy or polyneuropathy were accidentally included in this study. Third, the small sample size and the sex ratio being biased toward women are other limitations of this study. Large-scale studies with similar sex ratios are required for reaching a generalized conclusion. Fourth, the time spent on each technique was measured only during the performance of ultrasonography or NCS, not during preparation or setup. Therefore, the actual time required for STBFP may be different, according to individual laboratory settings. Finally, questionnaires rather than VAS scores may be suitable for assessing the discomfort of subjects during the NCS examination.

The stimulation between the PP and 2DPP sites produced larger amplitude SNAPs than the stimulation at other points in the LFCN conduction study. The STBFP could increase the response rate and ensure consistent procedure time of the LFCN conduction study, compared with the conventional technique. For clinical application, further studies are needed to evaluate the diagnostic role of STBFP in patients with meralgia paresthetica.

\section{CONFLICT OF INTEREST}

No potential conflict of interest relevant to this article were reported.

\section{ACKNOWLEDGMENTS}

This research was supported by a grant from the Institute of Clinical Medicine Research in Yeouido St. Mary's Hospital, Catholic University of Korea.

\section{AUTHOR CONTRIBUTION}

Conceptualization: Yoon MJ, Won SJ. Methodology: Yoon MJ, Park HM, Won SJ. Formal analysis: Yoon MJ, Won SJ. Project administration: Yoon MJ, Park HM, Won SJ. Visualization: Yoon MJ. Writing - original draft: Yoon MJ, Won SJ. Writing - review and editing: Yoon MJ, Won SJ. Approval of final manuscript: all authors.

\section{REFERENCES}

1. Tomaszewski KA, Popieluszko P, Henry BM, Roy J, Sanna B, Kijek MR, et al. The surgical anatomy of the lateral femoral cutaneous nerve in the inguinal region: a meta-analysis. Hernia 2016;20:649-57. 
2. Anloague PA, Huijbregts P. Anatomical variations of the lumbar plexus: a descriptive anatomy study with proposed clinical implications. J Man Manip Ther 2009;17:e107-14.

3. Ghent WR. Meralgia paraesthetica. Can Med Assoc J 1959;81:631-3.

4. Butler ET, Johnson EW, Kaye ZA. Normal conduction velocity in the lateral femoral cutaneous nerve. Arch Phys Med Rehabil 1974;55:31-2.

5. Spevak MK, Prevec TS. A noninvasive method of neurography in meralgia paraesthetica. Muscle Nerve 1995;18:601-5.

6. Boon AJ, Bailey PW, Smith J, Sorenson EJ, Harper CM, Hurdle MF. Utility of ultrasound-guided surface electrode placement in lateral femoral cutaneous nerve conduction studies. Muscle Nerve 2011;44:525-30.

7. Park BJ, Joeng ES, Choi JK, Kang S, Yoon JS, Yang SN. Ultrasound-guided lateral femoral cutaneous nerve conduction study. Ann Rehabil Med 2015;39:47-51.

8. Deimel GW, Hurst RW, Sorenson EJ, Boon AJ. Utility of ultrasound-guided near-nerve needle recording for lateral femoral cutaneous sensory nerve conduction study: does it increase reliability compared with surface recording? Muscle Nerve 2013;47:274-6.

9. Park GY, Im S, Lee JI, Lim SH, Ko YJ, Chung ME, et al. Effect of superficial peroneal nerve fascial penetration site on nerve conduction studies. Muscle Nerve 2010;41:227-33.
10. Park HJ, Yoon JS, Rhee WI, Kim JW, Yang SN, Won SJ. Effect of fascia penetration on sensory nerve conduction in the superficial radial nerve. J Clin Neurophysiol 2018;35:263-6.

11. Hanna A. The lateral femoral cutaneous nerve canal. J Neurosurg 2017;126:972-8.

12. Sarala PK, Nishihara T, Oh SJ. Meralgia paresthetica: electrophysiologic study. Arch Phys Med Rehabil 1979;60:30-1.

13. Jefferson D, Eames RA. Subclinical entrapment of the lateral femoral cutaneous nerve: an autopsy study. Muscle Nerve 1979;2:145-54.

14. Shin YB, Park JH, Kwon DR, Park BK. Variability in conduction of the lateral femoral cutaneous nerve. Muscle Nerve 2006;33:645-9.

15. Ray B, D'Souza AS, Kumar B, Marx C, Ghosh B, Gupta $\mathrm{NK}$, et al. Variations in the course and microanatomical study of the lateral femoral cutaneous nerve and its clinical importance. Clin Anat 2010;23:978-84.

16. Dias Filho LC, Valenca MM, Guimaraes Filho FA, Medeiros RC, Silva RA, Morais MG, et al. Lateral femoral cutaneous neuralgia: an anatomical insight. Clin Anat 2003;16:309-16.

17. Majkrzak A, Johnston J, Kacey D, Zeller J. Variability of the lateral femoral cutaneous nerve: an anatomic basis for planning safe surgical approaches. Clin Anat 2010;23:304-11. 